\title{
Optimal Quantization for Dyadic Homogeneous Cantor Distributions
}

\author{
Wolfgang Kreitmeier*1 \\ ${ }^{1}$ Fakultät für Informatik und Mathematik, Fachgebiet Maß- und Integrationstheorie, \\ Universität Passau, Innstraße 33, 94032 Passau, Germany.
}

Key words Quantization, homogeneous Cantor measures, Quantization dimension, Quantization coefficient. MSC (2000) 28A80

For a large class of dyadic homogeneous Cantor distributions in $\mathbb{R}$, which are not necessarily self-similar, we determine the optimal quantizers, give a characterization for the existence of the quantization dimension, and show the non-existence of the quantization coefficient. The class contains all self-similar dyadic Cantor distributions, with contraction factor less than or equal to $\frac{1}{3}$. For these distributions we calculate the quantization errors explicitly.

Copyright line will be provided by the publisher

\section{Introduction}

The problem of quantization originally arose in electrical engineering in the context of signal processing and data compression (cf. [5]). Mathematically the problem can be stated as follows. Given a Borel probability distribution $\nu$ on $\mathbb{R}^{d}, n \in \mathbb{N}=\{1,2, .$.$\} and r \geq 1$, determine the set

$$
C_{n, r}(\nu)=\left\{\beta \subset \mathbb{R}^{d}: \operatorname{card}(\beta) \leq n \text { and } \Psi_{\beta, r}(\nu)=V_{n, r}(\nu)\right\}
$$

of all $n$-optimal sets (quantizers) of order $r$, with

$$
\Psi_{\beta, r}(\nu)=\int \min _{b \in \beta}|x-b|^{r} d \nu(x)
$$

and

$$
V_{n, r}(\nu)=\inf _{\beta \subset \mathbb{R}^{d}, \operatorname{card}(\beta) \leq n} \Psi_{\beta, r}(\nu)
$$

the optimal quantization error. This problem of optimal quantization was systematically studied by Graf and Luschgy [3]. Under very general assumptions they have shown, that $C_{n, r}(\nu)$ is always non-empty (cf. [3], Theorem 4.12) and that every $\beta \in C_{n, r}(\nu)$ has exactly $n$ elements (cf. [3], Theorem 4.1).

The idea of defining a dimension $D_{r}$ by means of quantization goes back to Zador. In [11] he defined the quantization dimension

$$
D_{r}=\lim _{n \rightarrow \infty} \frac{r \log (n)}{-\log \left(V_{n, r}(\nu)\right)},
$$

if the limit exists. Later on this concept was investigated by several authors (see, for instance, [3], [8], [9]). If the quantization dimension $D_{r}$ exists, it can be asked whether the sequence $\left(n^{\frac{r}{D_{r}}} V_{n, r}(\nu)\right)_{n \in \mathbb{N}}$ converges, i.e. whether the quantization coefficient exists.

In the present paper we consider dyadic homogeneous Cantor distributions. To this end we first have to define dyadic homogeneous Cantor sets depending on a sequence $\left.\left.\left(c_{k}\right)_{k \in \mathbb{N}} \in\right] 0, \frac{1}{2}\right]^{\mathbb{N}}$ of contraction factors. The

\footnotetext{
* e-mail: wkreitmeier@gmx.de
} 
corresponding dyadic homogeneous Cantor measure is induced by the natural mass distribution on the dyadic homogeneous Cantor set and completely characterized through the sequence $\left(c_{k}\right)_{k \in \mathbb{N}}$. Recently, Kesseböhmer and Zhu [6] determined the optimal quantizers for these distributions, provided $\sup _{k \in \mathbb{N}} c_{k} \leq \frac{1}{4}$ (cf. [6], Proposition 3.7). Lindsay [7] was the first who used a special example of this type to show that the quantization dimension of order 1 need not to exist (cf. [7], Example 5.5). Later Kesseböhmer and Zhu [6] also gave such an example (cf. [6], Theorem $1.5(1))$.

If $c_{k}=c$ for every $k \in \mathbb{N}$ then the dyadic homogeneous Cantor distribution becomes self-similar. The special case $c=\frac{1}{3}$ yields the classical Cantor distribution. Under the additional assumption that $r=2$, Graf and Luschgy solved the quantization problem for this last distribution completely, i.e. they determined the optimal quantizers and the optimal quantization errors (cf. [2], Theorem 5.2). They proved the existence of the quantization dimension (cf. [2], Theorem 6.6) and that the quantization coefficient does not exist (cf. [2], Theorem 6.3).

As mentioned above, Lindsay was the first to study quantization for special non-self-similar Cantor measures. A general theory for the quantization of these measures was developed by Kesseböhmer and Zhu [6]. Many of their results are applicable to the classical Cantor Distribution ( cf. [6], Theorem 1.4, Theorem 1.6 (1) and (2) and Proposition 3.1). But their analysis of the $n$-optimal quantizers of order $r$ for dyadic homogeneous Cantor distributions is limited to $\sup _{k \in \mathbb{N}} c_{k} \leq \frac{1}{4}$ and, therefore, does not include the classical case.

We will generalize some of the results of Kesseböhmer and Zhu to the case that $\sup _{k \in \mathbb{N}} c_{k} \leq \frac{1}{3}$ (Theorem 4.4) and apply them to the classical Cantor distribution. Here we get new results in the case $r \neq 2$. Moreover, we will characterize those dyadic homogeneous Cantor distributions for which the quantization dimension exists (Remark 5.2). If the quantization dimension of the dyadic homogeneous Cantor distribution exists, we give necessary and sufficient conditions for the sequence $\left(n^{\frac{r}{D_{r}}} V_{n, r}(\nu)\right)_{n \in \mathbb{N}}$ to be bounded and bounded away from zero. In this situation the quantization coefficient does not exist (Proposition 5.3).

For the self similar case $\mu=\mu_{c}$ with $\left.\left.c \in\right] 0, \frac{1}{3}\right]$ we will derive a functional representation for $\underline{\lim }_{n \rightarrow \infty} n^{\frac{r}{D_{r}}} V_{n, r}\left(\mu_{c}\right)$ resp. $\varlimsup_{n \rightarrow \infty} n^{\frac{r}{D_{r}}} V_{n, r}\left(\mu_{c}\right)$. Such a characterization is not known for $c$ strictly between $\frac{1}{3}$ and $\frac{1}{2}$.

\section{Notation and basic facts}

First note that dyadic homogeneous Cantor sets are a special case of homogeneous Moran sets as proposed by Wen et.al. ( cf. [1], [10] ). Moreover, dyadic homogeneous Cantor sets and the related dyadic homogeneous Cantor distributions werde defined by Kesseböhmer and Zhu [6]. We follow the notation in [6] and will verify in this section some simple properties of these distributions.

Let $\left.\left.\left(c_{k}\right)_{k \in \mathbb{N}} \in\right] 0, \frac{1}{2}\right]^{\mathbb{N}}$ be given. The dyadic homogeneous Cantor set $C\left(\left(c_{k}\right)\right)$ is constructed inductively. Start with $E_{0}=[0,1]$ and let $\mathcal{D}_{0}=\left\{E_{0}\right\}$ be the collection of the so-called basic intervals of order 0 . Now remove an open interval of length $1-2 c_{1}$ in the middle of $E_{0}$, so that $\left[0, c_{1}\right]$ and $\left[1-c_{1}, 1\right]$ remain. We call the remaining sets, basic intervals of order 1 . The union of all basic intervals of order 1 is denoted by $E_{1}$, the collection of all these intervals by $\mathcal{D}_{1}$. Let $k \in \mathbb{N}$ and let $E_{k}$ be the union of all basic intervals of order $k$ and $\mathcal{D}_{k}$ the collection of all basic intervals of order $k$. Let $\pi_{k}=\prod_{i=1}^{k} c_{i}$. By removing an open interval of length $\left(1-2 c_{k+1}\right) \pi_{k}$ in the middle of each basic interval of order $k$ we obtain the collection $\mathcal{D}_{k+1}$ of basic intervals of order $k+1$, having all the length $\pi_{k+1}$. Set $C\left(\left(c_{k}\right)\right)=\bigcap_{k \in \mathbb{N}} E_{k}$.

The corresponding homogeneous Cantor measure $\mu$ is the unique Borel probability measure on $\mathbb{R}$, with

$$
\mu(F)=2^{-k} \text { for every } F \in \mathcal{D}_{k}
$$

and

$$
\mu(A)=\inf \left\{\sum_{i} \mu\left(U_{i}\right): A \cap C\left(\left(c_{k}\right)\right) \subset \bigcup_{i} U_{i}, U_{i} \in \bigcup_{k \geq 1} \mathcal{D}_{k}\right\}
$$

for every Borel subset $A \subset \mathbb{R}$. It has support $C\left(\left(c_{k}\right)\right)$. For the rest of this paper the distribution $\mu$ is always the dyadic homogeneous Cantor distribution defined by the sequence $\left(c_{k}\right)_{k \in \mathbb{N}}$ with $0<c_{k} \leq \frac{1}{3}$ for every $k \in \mathbb{N}$.

Let $\mathbb{N}_{0}=\{0,1,2, .$.$\} . For every k \in \mathbb{N}_{0}$ and $F \in \mathcal{D}_{k}$ we denote by $F_{1}$ the left and by $F_{2}$ the right basic interval of order $k+1$, contained in $F$. Moreover the midpoints of $F, F_{1}, F_{2}$ are denoted by $a, a_{1}, a_{2}$ resp. We 
make canonical use of this notation, for example $a_{121}$ denotes the midpoint of the basic interval $F_{121} \subset F$ of order $k+3$. For $t \in \mathbb{R}$ define

$$
\mathbb{R} \ni x \stackrel{\Theta_{t}}{\rightarrow} \Theta_{t}(x)=x+t
$$

as the translation by $t$. Moreover, let

$$
\mathbb{R} \ni x \stackrel{\Phi_{t}}{\rightarrow} \Phi_{t}(x)=t+(t-x)
$$

be the reflection relative to $t$. For an arbitrary Borel probability distribution $\nu$ on $\mathbb{R}$ and Borel measurable set $A \subset \mathbb{R}$ with $\nu(A)>0$, let $\nu(\cdot \mid A)=\frac{\nu(A \cap \cdot)}{\nu(A)}$ be the conditional distribution of $\nu$ w.r.t. A. Now we can state two simple but useful properties of $\mu$.

Lemma 2.1 Let $k \in \mathbb{N}_{0}$. Let $G, H \in \mathcal{D}_{k+2}$ with $\min (G) \leq \min (H)$. Let $t=\frac{1}{2}(\min (G)+\max (H))$ and $s=\min (H)-\min (G)$. Then

$$
\mu(\cdot \mid H)=\mu(\cdot \mid G) \circ \Phi_{t}^{-1}
$$

and

$$
\mu(\cdot \mid H)=\mu(\cdot \mid G) \circ \Theta_{s}^{-1} .
$$

Proof. Since $\operatorname{diam}(H)=\operatorname{diam}(G)$ we get from the definition of $t$ and $s$ that $\Phi_{t}(G)=H$ and $\Theta_{s}(G)=H$. Hence, the assertion follows from the construction of $\mu$.

\section{Elementary properties of optimal quantizers}

For the rest of this paper let $r \geq 1$ and let all distributions be defined on the Borel subsets of $\mathbb{R}$. Moreover a probability distribution $\nu$ is called symmetric with respect to $t \in \mathbb{R}$, if $\nu=\nu \circ \Phi_{t}^{-1}$.

We begin this section with a result about the 1-optimal quantizers of general symmetric distributions on $\mathbb{R}$. Then we use these statements to show elementary properties of the optimal quantizers of dyadic homogeneous Cantor measures.

Proposition 3.1 Let $\nu$ be a probability distribution which is symmetric w.r.t. $t \in \mathbb{R}$. Then for $r>1$ and $b \in \mathbb{R} \backslash\{t\}$,

$$
\int|x-b|^{r} d \nu(x)>\int|x-t|^{r} d \nu(x) .
$$

Proof. Let $b \in \mathbb{R} \backslash\{t\}$. From the proof of Theorem 2.4 in [3] we know that the mapping

$$
\mathbb{R} \ni z \rightarrow \Psi_{r}(z)=\int|x-z|^{r} d \nu(x)
$$

is strictly convex. Hence we have

$$
\int|x-t|^{r} d \nu(x)=\Psi_{r}(t)<\frac{\Psi_{r}(t+(b-t))+\Psi_{r}(t-(b-t))}{2} .
$$

Using the symmetry of $\nu$ w.r.t. $t$, we get $\Psi_{r}(t+(b-t))=\Psi_{r}(t-(b-t))$, which yields

$$
\frac{\Psi_{r}(t+(b-t))+\Psi_{r}(t-(b-t))}{2}=\Psi_{r}(b)=\int|x-b|^{r} d \nu(x)
$$

and completes the proof. 
Remark 3.2 If, in addition, $\nu$ has a compact support, then for $r=1$ the weaker inequality

$$
\int|x-b|^{r} d \nu(x) \geq \int|x-t|^{r} d \nu(x)
$$

still holds for all $b \in \mathbb{R}$.

Now fix $k \in \mathbb{N}_{0}$ and $F \in \mathcal{D}_{k}$ for the rest of this section.

Corollary 3.3 Let a be the midpoint of $F$. Then for $b \in \mathbb{R} \backslash\{a\}$,

$$
\int_{F}|x-b|^{r} d \mu(x) \geq \int_{F}|x-a|^{r} d \mu(x) .
$$

If $r>1$, then (3) is strict.

Proof. Since $\mu(F)>0$ it suffices to prove (3) for $\mu(\cdot \mid F)$ instead of $\mu$. Note, that $\mu(\cdot \mid F)$ is symmetric w.r.t. $a$ and has a compact support. Hence, the assertion follows directly from Proposition 3.1 and Remark 3.2.

Remark 3.4 Note, that the part for $r>1$ of Corollary 3.3 has already been proved in [6], Lemma 3.2.

For the rest of this section let $n \geq 2$ and $\beta \in C_{n, r}(\mu(\cdot \mid F))$. By [3], Theorem 4.1 we have $\operatorname{card}(\beta)=n$. Denote $\beta=\left\{b_{1}, . ., b_{n}\right\}$ with $b_{1}<. .<b_{n}$. Let $b_{0}=\Phi_{\min (F)}\left(b_{1}\right)$ and $b_{n+1}=\Phi_{\max (F)}\left(b_{n}\right)$. By [3], Remark 4.6 (a) we know, that $\beta \subset F$. Next we will prove some essential properties of $\beta$, in particular we will show, that $\beta \subset F_{1} \cup F_{2}$.

Lemma 3.5 Let $i \in\{1, . ., n\}$. If $\mu\left(\left[\frac{b_{i-1}+b_{i}}{2}, \frac{b_{i}+b_{i+1}}{2}\right] \cap F_{1}\right)=0$, then $b_{i} \in F_{2}$. If $\mu\left(\left[\frac{b_{i-1}+b_{i}}{2}, \frac{b_{i}+b_{i+1}}{2}\right] \cap F_{2}\right)=0$, then $b_{i} \in F_{1}$.

Proof. Since $\mu(\mathbb{R} \backslash[0,1])=0$, we get from [3], Theorem 4.1, that $\left[\frac{b_{i-1}+b_{i}}{2}, \frac{b_{i}+b_{i+1}}{2}\right]$ has strictly positive $\mu$-measure and $\left\{b_{i}\right\} \in C_{1, r}\left(\mu\left(\cdot \mid\left[\frac{b_{i-1}+b_{i}}{2}, \frac{b_{i}+b_{i+1}}{2}\right]\right)\right)$.

1. Let $\mu\left(\left[\frac{b_{i-1}+b_{i}}{2}, \frac{b_{i}+b_{i+1}}{2}\right] \cap F_{1}\right)=0$. Hence, $\mu\left(\cdot \mid\left[\frac{b_{i-1}+b_{i}}{2}, \frac{b_{i}+b_{i+1}}{2}\right]\right)$ is concentrated on $F_{2} \cap\left[\frac{b_{i-1}+b_{i}}{2}, \frac{b_{i}+b_{i+1}}{2}\right]$. Thus, Lemma 2.6 (a) in [3] yields $b_{i} \in F_{2}$.

2. As in 1 . we obtain $b_{i} \in F_{1}$, if $\mu\left(\left[\frac{b_{i-1}+b_{i}}{2}, \frac{b_{i}+b_{i+1}}{2}\right] \cap F_{2}\right)=0$.

Lemma 3.6 (a) $b_{1}$ belongs to $F_{1}$ and $b_{n}$ to $F_{2}$. (b) If $n \geq 3$, then

$$
1<j:=\min \left\{i \in\{1, \ldots, n\}: b_{i}>\max \left(F_{1}\right)\right\} \leq n
$$

and

(i) $b_{j-1} \leq \max \left(F_{1}\right)$,

(ii) $b_{j+1} \geq \min \left(F_{2}\right)$,

(iii) if $b_{j} \in F \backslash\left(F_{1} \cup F_{2}\right)$, then $j<n$ and $\frac{b_{j-1}+b_{j}}{2}<\max \left(F_{1}\right)$ and $\frac{b_{j}+b_{j+1}}{2}>\min \left(F_{2}\right)$.

Proof. By the construction of $\mu$ we can assume w.l.o.g. that $k=0$. Then we have $F=E_{0}=[0,1]$ and $\mu(\cdot \mid F)=\mu$. We proceed in several steps.

1. We will prove that $V_{2, r}(\mu) \leq 4 \int_{F_{11}}\left(\frac{c_{1}}{2}-x\right)^{r} d \mu(x)$.

By the symmetry of $\mu$ (Lemma 2.1) it is easy to see that

$$
V_{2, r}(\mu) \leq \Psi_{\left\{a_{1}, a_{2}\right\}, r}(\mu)=4 \int_{F_{11}}\left(\frac{c_{1}}{2}-x\right)^{r} d \mu(x) .
$$

2. We will show, that $b_{1}<\frac{3}{2} c_{1}$.

Assume the contrary, i.e. $b_{1} \geq \frac{3}{2} c_{1}$. This yields

$$
\begin{array}{r}
V_{2, r}(\mu) \geq V_{n, r}(\mu)=\Psi_{\beta, r}(\mu) \geq \int_{F_{1}}\left(b_{1}-x\right)^{r} d \mu(x) \geq \int_{F_{1}}\left(\frac{3}{2} c_{1}-x\right)^{r} d \mu(x) \\
=\int_{F_{11}}\left(\frac{3}{2} c_{1}-x\right)^{r} d \mu(x)+\int_{F_{12}}\left(\frac{3}{2} c_{1}-x\right)^{r} d \mu(x) .
\end{array}
$$


Note, that $\frac{3}{2} c_{1}-x \geq \frac{c_{1}}{2}$ for every $x \in F_{12}$ and $\frac{c_{1}}{2}-x \leq \frac{c_{1}}{2}$ for every $x \in F_{11}$, and, therefore,

$$
\int_{F_{12}}\left(\frac{3}{2} c_{1}-x\right)^{r} d \mu(x) \geq\left(\frac{c_{1}}{2}\right)^{r} \mu\left(F_{12}\right)=\left(\frac{c_{1}}{2}\right)^{r} \mu\left(F_{11}\right) \geq \int_{F_{11}}\left(\frac{c_{1}}{2}-x\right)^{r} d \mu(x) .
$$

Thus we get

$$
V_{2, r}(\mu) \geq \int_{F_{11}}\left(\frac{3}{2} c_{1}-x\right)^{r} d \mu(x)+\int_{F_{11}}\left(\frac{c_{1}}{2}-x\right)^{r} d \mu(x) .
$$

Since $r \geq 1$ it follows that for every $x \in F_{11}$,

$$
\left(\frac{3}{2} c_{1}-x\right)^{r}=3^{r}\left(\frac{c_{1}}{2}-\frac{1}{3} x\right)^{r} \geq 3\left(\frac{c_{1}}{2}-x\right)^{r} .
$$

Because the last inequality is strict for every $x \in F_{11} \backslash\{0\}$ we obtain

$$
V_{2, r}(\mu)>4 \int_{F_{11}}\left(\frac{c_{1}}{2}-x\right)^{r} d \mu(x),
$$

a contradiction to 1 .

3. We will show, that $b_{1} \leq c_{1}$.

Assume the contrary. By 2. we have

$$
c_{1}<b_{1}<\frac{3}{2} c_{1}
$$

If $\frac{b_{1}+b_{2}}{2}<1-c_{1}$, then Lemma 3.5 would imply $b_{1} \leq c_{1}$. Hence we have

$$
1-c_{1} \leq \frac{b_{1}+b_{2}}{2} \text {. }
$$

Combining (5) and (6) we get

$$
\begin{aligned}
b_{2} & \geq 2-2 c_{1}-b_{1}>2-\frac{7}{2} c_{1} \\
& =1-\frac{c_{1}}{2}+1-3 c_{1} \geq 1-\frac{c_{1}}{2} .
\end{aligned}
$$

We conclude that

$$
\begin{aligned}
& V_{2, r}(\mu) \geq \Psi_{\beta, r}(\mu) \geq \int_{F_{11}}\left(b_{1}-x\right)^{r} d \mu(x)+\int_{F_{12}}\left(b_{1}-x\right)^{r} d \mu(x) \\
& +\int_{F_{21} \cap\left[0, \frac{b_{1}+b_{2}}{2}\right]}\left(x-b_{1}\right)^{r} d \mu(x)+\int_{\left.\left.F_{21} \cap\right] \frac{b_{1}+b_{2}}{2}, 1\right]}\left(b_{2}-x\right)^{r} d \mu(x)
\end{aligned}
$$

Lemma 2.1 yields $\mu\left(\cdot \mid F_{21}\right)=\mu\left(\cdot \mid F_{11}\right) \circ \Theta_{1-c_{1}}^{-1}$ resp. $\mu\left(\cdot \mid F_{12}\right)=\mu\left(\cdot \mid F_{11}\right) \circ \Theta_{c_{1}\left(1-c_{2}\right)}^{-1}$. Thus, inequality (7) turns into

$$
\begin{aligned}
& V_{2, r}(\mu) \geq \int_{F_{11}}\left(b_{1}-x\right)^{r} d \mu(x)+\int_{F_{11}}\left(b_{1}-c_{1}\left(1-c_{2}\right)-x\right)^{r} d \mu(x) \\
& \quad+\int_{F_{11} \cap\left[0, \frac{b_{1}+b_{2}}{2}-\left(1-c_{1}\right)\right]}\left(x-\left(b_{1}-\left(1-c_{1}\right)\right)\right)^{r} d \mu(x) \\
& \quad+\int_{\left.F_{11} \cap\right]}\left(b_{2}-\left(1-c_{1}\right)-x\right)^{r} d \mu(x) .
\end{aligned}
$$


Let $x \in F_{11}$. Since $r \geq 1$ we have

$$
\begin{aligned}
& \frac{1}{2}\left[\left(b_{1}-c_{1}\left(1-c_{2}\right)-x\right)^{r}+\left(x-\left(b_{1}-\left(1-c_{1}\right)\right)\right)^{r}\right] \\
\geq & {\left[\frac{1}{2}\left(b_{1}-c_{1}+c_{1} c_{2}-x+x-b_{1}+1-c_{1}\right)\right]^{r} } \\
> & \left(\frac{1}{2}-c_{1}\right)^{r} \geq\left(\frac{c_{1}}{2}-x\right)^{r} .
\end{aligned}
$$

Using (6), we get similarly

$$
\begin{aligned}
& \frac{1}{2}\left[\left(b_{1}-c_{1}\left(1-c_{2}\right)-x\right)^{r}+\left(b_{2}-\left(1-c_{1}\right)-x\right)^{r}\right] \\
\geq & {\left[\frac{1}{2}\left(b_{1}-c_{1}+c_{1} c_{2}-x+b_{2}-1+c_{1}-x\right)\right]^{r} } \\
\geq & \left(\frac{b_{1}+b_{2}}{2}-\frac{1}{2}-x\right)^{r} \geq\left(1-c_{1}-\frac{1}{2}-x\right)^{r}
\end{aligned}
$$

Since $c_{1} \leq \frac{1}{3}$ we obtain

$$
\begin{aligned}
& \left(1-c_{1}-\frac{1}{2}-x\right)^{r} \\
= & \left(\frac{1}{2}-c_{1}-x\right)^{r} \geq\left(\frac{3}{2} c_{1}-c_{1}-x\right)^{r}=\left(\frac{c_{1}}{2}-x\right)^{r} .
\end{aligned}
$$

Using the combination of (9) and (10), inequality (8) turns into

$$
V_{2, r}(\mu) \geq \int_{F_{11}}\left(b_{1}-x\right)^{r} d \mu(x)+2 \int_{F_{11}}\left(\frac{c_{1}}{2}-x\right)^{r} d \mu(x) .
$$

Since $b_{1}>c_{1}$ and $c_{1}-x>c_{1}-2 x$ for every $x \in F_{11} \backslash\{0\}$ we deduce

$$
\begin{aligned}
& V_{2, r}(\mu) \geq \int_{F_{11}}\left(c_{1}-x\right)^{r} d \mu(x)+2 \int_{F_{11}}\left(\frac{c_{1}}{2}-x\right)^{r} d \mu(x) \\
> & 2^{r} \int_{F_{11}}\left(\frac{c_{1}}{2}-x\right)^{r} d \mu(x)+2 \int_{F_{11}}\left(\frac{c_{1}}{2}-x\right)^{r} d \mu(x) \geq 4 \int_{F_{11}}\left(\frac{c_{1}}{2}-x\right)^{r} d \mu(x),
\end{aligned}
$$

a contradiction to 1 .

4. We will proof that $b_{n} \geq 1-c_{1}$.

This follows from 3. by symmetry of $\mu$ (see Lemma 2.1).

5. We will finish the proof.

From 3. and 4. we get $b_{1} \in F_{1}, b_{n} \in F_{2}$ and $1<j \leq n$. If $n=2$, then $\beta \cap\left(F \backslash\left(F_{1} \cup F_{2}\right)\right)=\emptyset$.

Let $n \geq 3$. Clearly, $j<n$. From the definition of $j$ one gets $b_{j-1} \leq \max \left(F_{1}\right)$. If we assume $b_{j+1}<\min \left(F_{2}\right)$, Lemma 3.5 would imply $b_{j} \in F_{1}$, a contradiction to $b_{j}>\max \left(F_{1}\right)$. Hence, $b_{j+1} \geq \min \left(F_{2}\right)$. Finally let $b_{j} \in F \backslash\left(F_{1} \cup F_{2}\right)$. Due to $b_{n} \in F_{2}$ we have $j<n$.

Again, Lemma 3.5 yields $\frac{b_{j-1}+b_{j}}{2}<\max \left(F_{1}\right)$ and $\frac{b_{j}+b_{j+1}}{2}>\min \left(F_{2}\right)$.

Before we can continue to investigate the properties of $\beta$ we need a result for symmetric probability distributions, which is also interesting in itself.

Lemma 3.7 Let $r>1, t \in \mathbb{R}$ and let $\nu$ be a non-atomic probability distribution which is symmetric w.r.t. $t \in \mathbb{R}$. Let $\{a, b, c\} \subset \mathbb{R}$ with $a<b=\Phi_{t}(c)<t<c$. If $[a, c]$ has positive $\nu$-measure, then every element of an $1-$ optimal quantizer of $\nu(\cdot \mid[a, c])$ is smaller or equal to $t$. Equality only holds, if $\nu([a, b])=0$. 
Proof. W.l.o.g. we may assume $t=0$. Let $r>1$ and $\left\{t^{\prime}\right\} \in C_{1, r}(\nu(\cdot \mid[a, c]))$.

1. We will show, that $t^{\prime}=0$, if and only if $\nu([a, b])=0$.

Let $t^{\prime}=0$. Since $r>1$, one gets from [3], Lemma 2.5, that

$$
0=-\int_{[a, 0[}|x|^{r-1} d \nu(x)+\int_{] 0, c]}|x|^{r-1} d \nu(x) .
$$

Due to $\nu(\{z\})=0$ for every $z \in \mathbb{R}$ and the symmetry of $\nu$ w.r.t. 0 we derive

$$
\begin{aligned}
0 & =\int_{[a, b]}|x|^{r-1} d \nu(x)+\int_{\left[\Phi_{0}(c), 0\right]}|x|^{r-1} d \nu(x)-\int_{[0, c]}|x|^{r-1} d \nu(x) \\
& =\int_{[a, b]}|x|^{r-1} d \nu(x) \geq \nu([a, b]) \cdot|b|^{r-1},
\end{aligned}
$$

which yields $\nu([a, b])=0$. On the other hand, if $\nu([a, b])=0$, we get $\nu([a, c])=\nu([b, c])$. By Proposition 3.1 we derive $t^{\prime}=0$.

2. We will show, that $t^{\prime} \leq 0$.

Assume the contrary. From the symmetry of $\nu$ and Proposition 3.1 we get

$$
\begin{aligned}
\int_{[a, c]}\left|x-t^{\prime}\right|^{r} d \nu(x) & =\int_{[a, b]}\left|x-t^{\prime}\right|^{r} d \nu(x)+\int_{[b, c]}\left|x-t^{\prime}\right|^{r} d \nu(x) \\
& >\int_{[a, b]}\left|x-t^{\prime}\right|^{r} d \nu(x)+\int_{[b, c]}|x|^{r} d \nu(x) .
\end{aligned}
$$

Due to $t^{\prime}>0$ and $b<0$ we have for every $x \in[a, b]$ that $\left|x-t^{\prime}\right|>|x|$. This yields

$$
\int_{[a, c]}\left|x-t^{\prime}\right|^{r} d \nu(x)>\int_{[a, c]}|x|^{r} d \nu(x),
$$

a contradiction to the optimality of $t^{\prime}$.

Remark 3.8 By the same arguments as in the proof of Lemma 3.7 one can show for $b=\Phi_{t}(c)<a<t<c$, that every element of an 1 -optimal set of $\nu(\cdot \mid[a, c])$ is greater or equal to $t$, provided $\nu([a, c])>0$. Moreover, equality holds in this case if and only if $\nu([b, a])=0$.

Now we continue with a result for the 3 -optimal quantizers of $\mu(\cdot \mid F)$. We denote the midpoint of $F$ by $a$.

Lemma 3.9 Let $r>1$ and $n=3$. If $b_{2} \in\left[a, \max (F)-\pi_{k+1}[\right.$ then

$$
\max \left(F_{1}\right)-\frac{b_{1}+b_{2}}{2} \leq \frac{b_{2}+b_{3}}{2}-\min \left(F_{2}\right) .
$$

Proof. By the construction of $\mu$ we can assume w.l.o.g. that $F=[0,1]$. Thus we have $\max \left(F_{1}\right)=c_{1}$ and $\min \left(F_{2}\right)=1-c_{1}$. Assume that

$$
c_{1}-\frac{b_{1}+b_{2}}{2}>\frac{b_{2}+b_{3}}{2}-\left(1-c_{1}\right) \text {. }
$$

We will show, that $b_{2}<\frac{1}{2}=a$. From (11) we obtain $\frac{b_{1}+b_{2}}{2}<1-\frac{b_{2}+b_{3}}{2}=\Phi_{\frac{1}{2}}\left(\frac{b_{2}+b_{3}}{2}\right)$. From the construction of $\mu$ we know, that $\mu$ is non-atomic. By Lemma 3.6 (b) (iii) we obtain $\frac{b_{2}+b_{3}}{2}>1-c_{1}>\frac{1}{2}$, which yields $\Phi_{\frac{1}{2}}\left(\frac{b_{2}+b_{3}}{2}\right)<\frac{1}{2}$. Since

$$
\mu=\mu(\cdot \mid F)=\frac{1}{4}\left[\mu\left(\cdot \mid F_{11}\right)+\mu\left(\cdot \mid F_{12}\right)+\mu\left(\cdot \mid F_{21}\right)+\mu\left(\cdot \mid F_{22}\right)\right],
$$

Lemma 2.1 yields $\mu \circ \Phi_{\frac{1}{2}}^{-1}=\mu$ i.e. $\mu$ is symmetric w.r.t. $\frac{1}{2}$. By [3], Theorem 4.1 we know, that $\mu\left(\left[\frac{b_{1}+b_{2}}{2}, \frac{b_{2}+b_{3}}{2}\right]\right)>0$ and $\left\{b_{2}\right\}$ is an 1-optimal quantizer of order $r$ for $\mu\left(\cdot \mid\left[\frac{b_{1}+b_{2}}{2}, \frac{b_{2}+b_{3}}{2}\right]\right)$. By Lemma 3.7, 
we have $b_{2} \leq \frac{1}{2}$. It remains to show, that the assumption $b_{2}=\frac{1}{2}$ leads to a contradiction. Assume $b_{2}=\frac{1}{2}$. Since $r>1$, we know from [3], Theorem 2.4 and Theorem 4.1, that $\left\{b_{1}\right\}$ is the unique 1 -optimal quantizer of $\mu\left(\cdot \mid\left[0, \frac{b_{1}+b_{2}}{2}\right]\right)$ resp. $\left\{b_{3}\right\}$ is the unique 1 -optimal quantizer of $\mu\left(\cdot \mid\left[\frac{b_{2}+b_{3}}{2}, 1\right]\right)$. By Lemma 3.7 we have $\mu\left(\cdot \mid\left[\frac{b_{1}+b_{2}}{2}, \Phi_{\frac{1}{2}}\left(\frac{b_{2}+b_{3}}{2}\right)\right]\right)=0$,

which yields $\mu\left(\cdot \mid\left[0, \frac{b_{1}+b_{2}}{2}\right]\right)=\mu\left(\cdot \mid\left[0, \Phi_{\frac{1}{2}}\left(\frac{b_{2}+b_{3}}{2}\right)\right]\right)$. Using the symmetry of $\mu$ we get from [3], Lemma 2.1 (a), that $\Phi_{\frac{1}{2}}\left(b_{1}\right)=b_{3}$. Hence, $b_{2}-b_{1}=b_{3}-b_{2}$, which yields $c_{1}-\frac{b_{1}+b_{2}}{2}=\frac{b_{2}+b_{3}}{2}-\left(1-c_{1}\right)$, a contradiction to inequality (11).

Before we can state the main result of this section, we need the following inequality.

Lemma 3.10 Let $A, B, C$ and $D$ be non-negative real numbers. If $A+B>C+D$ and $\max (A, B)>\max (C, D)$, then

$$
A^{r}+B^{r}>C^{r}+D^{r}
$$

Pro of. Immediate consequence of the convexity of $s \mapsto s^{r}$.

Proposition 3.11 For $r>1$ the set $\beta$ is contained in $F_{1} \cup F_{2}$ and, moreover, $\beta \cap F_{1} \neq \emptyset$ and $\beta \cap F_{2} \neq \emptyset$.

Proof. Again, we can assume w.l.o.g. that $k=0$ and, therefore, $F=[0,1]$. Recall, that $\beta=\left\{b_{1}, . ., b_{n}\right\}$ with $b_{1}<. .<b_{n}$. Lemma 3.6 (b) yields $\beta \cap F_{1} \neq \emptyset, \beta \cap F_{2} \neq \emptyset$ and $\operatorname{card}\left(\beta \cap\left(F \backslash\left(F_{1} \cup F_{2}\right)\right)\right) \leq 1$. It remains to show, that $\beta \cap\left(F \backslash\left(F_{1} \cup F_{2}\right)\right)=\emptyset$.

Case 1. $n=2$

In this case the assertion follows from Lemma 3.6 (a).

Case 2. $n=3$

We will prove the assertion by contradiction. Assume, that $\beta \cap\left(F \backslash\left(F_{1} \cup F_{2}\right)\right) \neq \emptyset$. By Lemma 3.5, $b_{2}$ is the only element of $\beta \cap\left(F \backslash\left(F_{1} \cup F_{2}\right)\right)$.

Since $\mu$ is symmetric w.r.t. $\frac{1}{2}$ we can assume that w.l.o.g. $b_{2} \geq \frac{1}{2}$.

First, we will prove that for every $x \in F_{11}$ we have

$$
\min _{i=1,2}\left|c_{1}-x-b_{i}\right|^{r}+\min _{i=2,3}\left|1-c_{1}+x-b_{i}\right|^{r} \geq 2\left(\frac{c_{1}}{2}-x\right)^{r}
$$

By Lemma 3.6 (b) (iii) and Lemma 3.9 we obtain

$$
0<c_{1}-\frac{b_{1}+b_{2}}{2} \leq \frac{b_{2}+b_{3}}{2}-\left(1-c_{1}\right) .
$$

Let $x \in F_{11}$ with $0 \leq x \leq c_{1}-\frac{b_{1}+b_{2}}{2}$. Since $c_{1}-x \geq \frac{b_{1}+b_{2}}{2}$ we get

$$
\min _{i=1,2}\left|c_{1}-x-b_{i}\right|=b_{2}-c_{1}+x \text {. }
$$

Using (13) we have $1-c_{1}+x \leq 1-c_{1}+\frac{b_{2}+b_{3}}{2}-\left(1-c_{1}\right)=\frac{b_{2}+b_{3}}{2}$, which yields

$$
\min _{i=2,3}\left|1-c_{1}+x-b_{i}\right|=1-c_{1}+x-b_{2} .
$$

Since $r \geq 1$ the equalities (14) and (15) together with $x \leq \frac{c_{1}}{2}$ imply that

$$
\begin{aligned}
& \min _{i=1,2}\left|c_{1}-x-b_{i}\right|^{r}+\min _{i=2,3}\left|1-c_{1}+x-b_{i}\right|^{r} \\
= & \left(b_{2}-c_{1}+x\right)^{r}+\left(1-c_{1}+x-b_{2}\right)^{r} \\
\geq & 2\left(\frac{1}{2}\left(b_{2}-c_{1}+x+1-c_{1}+x-b_{2}\right)\right)^{r} \\
= & 2\left(\frac{1}{2}\left(1-2 c_{1}+2 x\right)\right)^{r} \\
\geq & 2\left(\frac{c_{1}}{2}+x\right)^{r} \geq 2\left(\frac{c_{1}}{2}-x\right)^{r} .
\end{aligned}
$$


Now let $x \in F_{11}$ with $c_{1}-\frac{b_{1}+b_{2}}{2} \leq x \leq \frac{b_{2}+b_{3}}{2}-\left(1-c_{1}\right)$. We have $c_{1}-x \leq \frac{b_{1}+b_{2}}{2}$. By Lemma 3.6 (b) (iii) we get $\frac{b_{1}+b_{2}}{2}<c_{1}$, which yields

$$
b_{1}<2 c_{1}-b_{2} \leq 2 c_{1}-\frac{1}{2} \leq \frac{c_{1}}{2},
$$

and, therefore

$$
b_{1}<\frac{c_{1}}{2}<\frac{2}{3} c_{1} \leq c_{1}\left(1-c_{2}\right) \leq c_{1}-x .
$$

Hence we obtain

$$
\min _{i=1,2}\left|c_{1}-x-b_{i}\right|=c_{1}-x-b_{1} .
$$

On the other hand, $1-c_{1}+x \leq \frac{b_{2}+b_{3}}{2}$. Hence, equation (15) holds in this case as well. Combining (15) and (17) we obtain

$$
\begin{gathered}
\min _{i=1,2}\left|c_{1}-x-b_{i}\right|^{r}+\min _{i=2,3}\left|1-c_{1}+x-b_{i}\right|^{r} \\
\left.\geq 2\left(\frac{1}{2}\left(1-b_{1}-b_{2}\right)\right)^{r}=2\left(\frac{1}{2}-\frac{b_{1}+b_{2}}{2}\right)\right)^{r} .
\end{gathered}
$$

Recall, that $x \leq \max \left(F_{11}\right)<\frac{c_{1}}{2}$. Together with $\frac{b_{1}+b_{2}}{2}<c_{1}$ and $\frac{3}{2} c_{1} \leq \frac{1}{2}$ we get

$$
\begin{array}{r}
\min _{i=1,2}\left|c_{1}-x-b_{i}\right|^{r}+\min _{i=2,3}\left|1-c_{1}+x-b_{i}\right|^{r} \\
\left.\left.\left.>2\left(\frac{3}{2} c_{1}-c_{1}\right)\right)^{r}=2\left(\frac{c_{1}}{2}\right)\right)^{r} \geq 2\left(\frac{c_{1}}{2}-x\right)\right)^{r}
\end{array}
$$

Finally let $x \in F_{11}$ and $\frac{b_{2}+b_{3}}{2}-\left(1-c_{1}\right)<x$. Obviously, equation (17) also holds in this case. Since $r>1$, we know from [3], Theorem 2.4 and Theorem 4.1, that $\mu\left(\left[\frac{b_{2}+b_{3}}{2}, 1\right]\right)>0$ and $\left\{b_{3}\right\}$ is the unique $1-$ optimal quantizer of $\mu\left(\cdot \mid\left[\frac{b_{2}+b_{3}}{2}, 1\right]\right)$. Since $\mu\left(\cdot \mid\left[1-c_{1}, 1\right]\right)=\frac{1}{2}\left[\mu\left(\cdot \mid F_{21}\right)+\mu\left(\cdot \mid F_{22}\right)\right]$ we obtain from Lemma 2.1 that $\mu\left(\cdot \mid\left[1-c_{1}, 1\right]\right)$ is symmetric w.r.t. $1-\frac{c_{1}}{2}$. Note, that $\mu\left(\cdot \mid\left[1-c_{1}, 1\right]\right)$ is non-atomic. Moreover, $\mu\left(\left[1-c_{1}, \frac{b_{2}+b_{3}}{2}\right]\right)>0$, because otherwise Lemma 3.5 would imply $b_{2} \in F_{1}$, a contradiction. By Remark 3.8 we get $b_{3}>1-\frac{c_{1}}{2}$. Therefore,

$$
b_{3}>1-\frac{c_{1}}{2}>1-\frac{2}{3} c_{1} \geq 1-c_{1}+c_{1} c_{2}=1-c_{1}+\max \left(F_{11}\right) \geq 1-c_{1}+x .
$$

Hence it follows from (17) that

$$
\min _{i=2,3}\left|1-c_{1}+x-b_{i}\right|=b_{3}-\left(1-c_{1}+x\right) .
$$

Using (17) and (20) we derive

$$
\begin{aligned}
\min _{i=1,2} \mid c_{1} & -x-\left.b_{i}\right|^{r}+\min _{i=2,3}\left|1-c_{1}+x-b_{i}\right|^{r} \\
& \geq 2\left(\frac{1}{2}\left(c_{1}-b_{1}-\left(1-b_{3}\right)+c_{1}-2 x\right)\right)^{r} .
\end{aligned}
$$

Using (16) we get $c_{1}-b_{1}>\frac{c_{1}}{2}$. By (19) we have $1-b_{3}<\frac{c_{1}}{2}$. We obtain for every $x \in F_{11}$

$$
\left.\min _{i=1,2}\left|c_{1}-x-b_{i}\right|^{r}+\min _{i=2,3}\left|1-c_{1}+x-b_{i}\right|^{r}>2\left(\frac{c_{1}}{2}-x\right)\right)^{r}
$$

and (12) is proved. Since $\max \left(F_{11}\right)=c_{1} c_{2}<\frac{1}{4} \leq \frac{b_{1}+b_{2}}{2}<\min \left(F_{2}\right)$ and $\min \left(F_{2}\right)<\frac{b_{2}+b_{3}}{2}<\frac{\left(1-c_{1}\right)+1}{2}<1-c_{1} c_{2}=\min \left(F_{22}\right)$ we deduce

$$
\begin{aligned}
V_{3, r}(\mu) & =\int_{F_{11}}\left|x-b_{1}\right|^{r} d \mu(x)+\int_{F_{12}} \min _{i=1,2}\left|x-b_{i}\right|^{r} d \mu(x) \\
& +\int_{F_{21}} \min _{i=2,3}\left|x-b_{i}\right|^{r} d \mu(x)+\int_{F_{22}}\left|x-b_{3}\right|^{r} d \mu(x)
\end{aligned}
$$


Applying Lemma 2.1, we get

$$
\begin{aligned}
V_{3, r}(\mu) & =\int_{F_{11}}\left|x-b_{1}\right|^{r} d \mu(x)+\int_{\Phi_{\frac{c_{1}}{2}}^{-1}\left(F_{12}\right)} \min _{i=1,2}\left|\Phi_{\frac{c_{1}}{2}}(x)-b_{i}\right|^{r} d \mu(x) \\
& +\int_{\Phi_{\frac{c_{1}}{2}}^{-1} \Phi_{\frac{1}{2}}^{-1}\left(F_{21}\right)} \min _{i=2,3}\left|\Phi_{\frac{1}{2}} \circ \Phi_{\frac{c_{1}}{2}}(x)-b_{i}\right|^{r} d \mu(x)+\int_{F_{22}}\left|x-b_{3}\right|^{r} d \mu(x) \\
& =\int_{F_{11}}\left|x-b_{1}\right|^{r} d \mu(x)+\int_{F_{22}}\left|x-b_{3}\right|^{r} d \mu(x) \\
& +\int_{F_{11}} \min _{i=1,2}\left|c_{1}-x-b_{i}\right|^{r} d \mu(x)+\int_{F_{11}} \min _{i=2,3}\left|1-c_{1}+x-b_{i}\right|^{r} d \mu(x) .
\end{aligned}
$$

Corollary 3.3 yields

$$
\begin{array}{r}
V_{3, r}(\mu) \geq \int_{F_{11}}\left|x-a_{11}\right|^{r} d \mu(x)+\int_{F_{22}}\left|x-a_{22}\right|^{r} d \mu(x) \\
+\int_{F_{11}} \min _{i=1,2}\left|c_{1}-x-b_{i}\right|^{r} d \mu(x)+\int_{F_{11}} \min _{i=2,3}\left|1-c_{1}+x-b_{i}\right|^{r} d \mu(x) .
\end{array}
$$

We know, that inequality (12) is strict on a subset of $F_{11}$ with positive $\mu$ measure. Hence we obtain

$$
V_{3, r}(\mu)>\int_{F_{11}}\left|x-a_{11}\right|^{r} d \mu(x)+\int_{F_{22}}\left|x-a_{22}\right|^{r} d \mu(x)+2 \int_{F_{11}}\left(\frac{c_{1}}{2}-x\right)^{r} d \mu(x) .
$$

From Lemma 2.1 we get $\int_{F_{11}}\left|x-a_{11}\right|^{r} d \mu(x)=\int_{F_{21}}\left|x-a_{21}\right|^{r} d \mu(x)$ resp. $\int_{F_{11}}\left(\frac{c_{1}}{2}-x\right)^{r} d \mu(x)=\int_{F_{12}}\left(x-\frac{c_{1}}{2}\right)^{r} d \mu(x)$. Thus we conclude

$$
\begin{aligned}
V_{3, r}(\mu) & >\int_{F_{21}}\left|x-a_{21}\right|^{r} d \mu(x)+\int_{F_{22}}\left|x-a_{22}\right|^{r} d \mu(x) \\
& +\int_{F_{11}}\left(\frac{c_{1}}{2}-x\right)^{r} d \mu(x)+\int_{F_{12}}\left(x-\frac{c_{1}}{2}\right)^{r} d \mu(x) .
\end{aligned}
$$

By $a_{1}=\frac{c_{1}}{2}$ we have $\int_{F_{11}}\left(\frac{c_{1}}{2}-x\right)^{r} d \mu(x)+\int_{F_{12}}\left(x-\frac{c_{1}}{2}\right)^{r} d \mu(x)=\int_{F_{1}}\left|x-a_{1}\right|^{r} d \mu(x)$. Hence we deduce

$$
\begin{aligned}
V_{3, r}(\mu) & >\int_{F_{21}}\left|x-a_{21}\right|^{r} d \mu(x)+\int_{F_{22}}\left|x-a_{22}\right|^{r} d \mu(x)+\int_{F_{1}}\left|x-a_{1}\right|^{r} d \mu(x) \\
& =\Psi_{\left\{a_{1}, a_{21}, a_{22}\right\}}(\mu) \geq V_{3, r}(\mu),
\end{aligned}
$$

a contradiction.

Case 3. $n \geq 4$

We will prove the assertion by contradiction.

Assume that $\beta \cap\left(F \backslash\left(F_{1} \cup F_{2}\right)\right) \neq \emptyset$. By Lemma 3.6 we have $\left\{b_{j}\right\}=\beta \cap\left(F \backslash\left(F_{1} \cup F_{2}\right)\right)$ with $j$ defined in (4) and $\frac{b_{j-1}+b_{j}}{2}<c_{1}$. Let $\alpha=\left\{a_{11}, a_{12}, a_{21}, a_{22}\right\}$ be the set of midpoints of the sets $F_{11}, F_{12}, F_{21}$ and $F_{22}$ resp. By the symmetry of $\mu$ (Lemma 2.1) we get

$$
\begin{aligned}
& \int_{F} \min _{a \in \alpha}|x-a|^{r} d \mu(x) \\
& =\int_{F_{11} \cup F_{12} \cup F_{21} \cup F_{22}} \min _{a \in \alpha}|x-a|^{r} d \mu(x) \\
& =4 \int_{F_{11}}\left|x-a_{11}\right|^{r} d \mu(x) \\
& =4 \int_{F_{1111} \cup F_{1112} \cup F_{1121} \cup F_{1122}}\left|x-a_{11}\right|^{r} d \mu(x) .
\end{aligned}
$$


Since $a_{11}=\frac{\pi_{2}}{2}$ we obtain

$$
\max _{x \in F_{1111}}\left|x-a_{11}\right|=\frac{\pi_{2}}{2}=\max _{x \in F_{1122}}\left|x-a_{11}\right|
$$

and

$$
\max _{x \in F_{1112}}\left|x-a_{11}\right|=\frac{\pi_{2}}{2}-\pi_{3}+\pi_{4}=\max _{x \in F_{1121}}\left|x-a_{11}\right| .
$$

Combining (22), (23) and (24) we get

$$
\begin{aligned}
& \int_{F} \min _{a \in \alpha}|x-a|^{r} d \mu(x) \\
& \leq 4 \cdot 2 \mu\left(F_{1111}\right)\left(\left(\frac{\pi_{2}}{2}\right)^{r}+\left(\frac{\pi_{2}}{2}-\pi_{3}+\pi_{4}\right)^{r}\right) \\
& =\frac{c_{1}^{r}}{2}\left(\left(\frac{c_{2}}{2}\right)^{r}+\left(\frac{c_{2}}{2}-c_{2} c_{3}+c_{2} c_{3} c_{4}\right)^{r}\right) \\
& \leq \frac{c_{1}^{r}}{4}\left(c_{2}^{r}+\left(c_{2}-2 c_{2} c_{3}+2 c_{2} c_{3} c_{4}\right)^{r}\right) .
\end{aligned}
$$

Let $x \in F_{122}$. If $\frac{b_{j-1}+b_{j}}{2} \notin F_{122}$ we have

$$
\min _{b \in \beta}|x-b|=b_{j}-x \geq b_{j}-c_{1} \geq \max \left(0, b_{j}-c_{1}-\pi_{3}\right) .
$$

If $\frac{b_{j-1}+b_{j}}{2} \in F_{122}$ and $b_{j}-c_{1}>\pi_{3}$, then, $b_{j-1}<\min F_{122}$ and

$$
\min _{b \in \beta}|x-b| \geq \min \left(b_{j}-x, x-b_{j-1}\right) \geq \min \left(b_{j}-c_{1}, c_{1}-\pi_{3}-b_{j-1}\right)
$$

Since $c_{1} \geq \frac{1}{2}\left(b_{j-1}+b_{j}\right)$ and therefore $c_{1}-b_{j-1} \geq b_{j}-c_{1}$, we obtain

$$
\min \left(b_{j}-c_{1}, c_{1}-\pi_{3}-b_{j-1}\right) \geq \max \left(0, b_{j}-c_{1}-\pi_{3}\right) .
$$

If $b_{j}-c_{1} \leq \pi_{3}$, then

$$
\min _{b \in \beta}|x-b| \geq 0=\max \left(0, b_{j}-c_{1}-\pi_{3}\right) .
$$

Combining these inequalities we obtain

$$
\min _{x \in F_{122}} \min _{b \in \beta}|x-b| \geq \max \left(0, b_{j}-c_{1}-\pi_{3}\right) .
$$

Similarly one derives

$$
\begin{aligned}
& \min _{x \in F_{211}} \min _{b \in \beta}|x-b| \geq \max \left(0,1-c_{1}-b_{j}-\pi_{3}\right), \\
& \min _{x \in F_{121}} \min _{b \in \beta}|x-b| \geq \max \left(0, b_{j}-c_{1}-\pi_{2}\right),
\end{aligned}
$$

and

$$
\min _{x \in F_{212}} \min _{b \in \beta}|x-b| \geq \max \left(0,1-c_{1}-b_{j}-\pi_{2}\right) .
$$

Combining (26), (27), (28), and (29) we calculate

$$
\begin{aligned}
V_{n, r}(\mu)= & \int_{F} \min _{b \in \beta}|x-b|^{r} d \mu(x) \\
> & \int_{F_{121} \cup F_{122} \cup F_{211} \cup F_{212}} \min _{b \in \beta}|x-b|^{r} d \mu(x) \\
\geq & \frac{1}{8}\left(\left(\max \left(0, b_{j}-c_{1}-\pi_{3}\right)\right)^{r}+\left(\max \left(0,1-c_{1}-b_{j}-\pi_{3}\right)\right)^{r}\right) \\
& +\frac{1}{8}\left(\left(\max \left(0, b_{j}-c_{1}-\pi_{2}\right)\right)^{r}+\left(\max \left(0,1-c_{1}-b_{j}-\pi_{2}\right)\right)^{r}\right) .
\end{aligned}
$$


Due to $\sup _{j \in \mathbb{N}} c_{j} \leq \frac{1}{3}$ we get

$$
\begin{aligned}
& V_{n, r}(\mu)>\frac{1}{8}\left(2\left(\frac{1-2 c_{1}-2 \pi_{3}}{2}\right)^{r}+2\left(\frac{1-2 c_{1}-2 \pi_{2}}{2}\right)^{r}\right) \\
& \geq \frac{c_{1}^{r}}{4}\left(\left(\frac{1}{2}-c_{2} c_{3}\right)^{r}+\left(\frac{1}{2}-c_{2}\right)^{r}\right) .
\end{aligned}
$$

Now let $A=\frac{1}{2}-c_{2} c_{3}$ and $B=\frac{1}{2}-c_{2}$, resp. $C=c_{2}$ and $D=c_{2}-2 c_{2} c_{3}+2 c_{2} c_{3} c_{4}$. Note, that $A, B, C$ and $D$ are non-negative real numbers. Moreover,

$$
\begin{aligned}
& A+B-(C+D) \\
= & \left(\frac{1}{2}-c_{2} c_{3}\right)+\left(\frac{1}{2}-c_{2}\right)-\left(c_{2}+\left(c_{2}-2 c_{2} c_{3}+2 c_{2} c_{3} c_{4}\right)\right) \\
= & 1-c_{2}\left(3+c_{3}\left(2 c_{4}-1\right)\right)>1-3 c_{2} \geq 0,
\end{aligned}
$$

and $\max (A, B)=A>C=\max (C, D)$. By Lemma 3.10 we get

$$
\left(\frac{1}{2}-c_{2} c_{3}\right)^{r}+\left(\frac{1}{2}-c_{2}\right)^{r}>c_{2}^{r}+\left(c_{2}-2 c_{2} c_{3}+2 c_{2} c_{3} c_{4}\right)^{r} .
$$

Combining the last inequality with (30) and (25) we obtain

$$
\int_{F} \min _{a \in \alpha}|x-a|^{r} d \mu(x)<\int_{F} \min _{b \in \beta}|x-b|^{r} d \mu(x),
$$

which contradicts the optimality of $\beta$.

Hence in all cases we have $\beta \cap\left(F \backslash\left(F_{1} \cup F_{2}\right)\right)=\emptyset$ and the proof of Proposition 3.11 is complete.

Remark 3.12 By checking all possibilities, it is easy to see, that

$$
\min _{i=1,2}\left|c_{1}-x-b_{i}\right|+\min _{i=2,3}\left|1-c_{1}+x-b_{i}\right| \geq c_{1}-2 x
$$

holds for every $x \in F_{11}$. Moreover, all other arguments in the proof of Proposition 3.11 (especially the application of Corollary 3.3) also work for $r=1$. Hence, Proposition 3.11 is also valid for $r=1$.

\section{Determination of the optimal quantizers and the optimal quantization error}

In this section we will determine the $n$-optimal quantizers for $\mu$ and derive a formula for the quantization error. To this end, we parallel the approach in the proofs of Lemma 3.3 - 3.6 and Proposition 3.7 in [6], making amendments to the case $\sup _{j \in \mathbb{N}} c_{j} \leq \frac{1}{3}$ where necessary.

For a finite non-empty set $\alpha \subset \mathbb{R}$ and $a \in \alpha$ let

$$
W(a \mid \alpha)=\left\{x \in \mathbb{R}:|x-a|=\min _{b \in \alpha}|x-b|\right\}
$$

be the Voronoi cell generated by $a \in \alpha$. In this section $\beta$ denotes an arbitrary finite non-empty subset of $\mathbb{R}$.

Lemma 4.1 Let $k \in \mathbb{N}$ and $\beta \subset E_{k-1}$. Let $F \in \mathcal{D}_{k-1}$ with $\beta \cap F \neq \emptyset$. Then

$$
\min _{b \in \beta \cap F}|x-b|=\min _{b \in \beta}|x-b|
$$

holds for every $x \in F$. If, in addition, $\beta \cap H \neq \emptyset$ for every $H \in \mathcal{D}_{k-1}$, then $\mu\left(\cdot \mid \bigcup_{b \in \beta \cap F} W(b \mid \beta)\right)=\mu(\cdot \mid F)$. 
Proof. Equation (32) holds since $\sup _{j \in \mathbb{N}} c_{j} \leq \frac{1}{3} \operatorname{implies} \min _{x \in F, y \in H}|x-y| \geq \operatorname{diam}(F)$ for every $F, H \in \mathcal{D}_{k-1}$ with $F \neq H$.

Now assume $\beta \cap H \neq \emptyset$ for every $H \in \mathcal{D}_{k-1}$. Combining (31) with (32) we know, that $F$ is a subset of $\bigcup_{b \in \beta \cap F} W(b \mid \beta)$. Since $\sup _{j \in \mathbb{N}} c_{j} \leq \frac{1}{3}$ and $\mu$ is non-atomic, we get $\mu\left(H \cap \bigcup_{b \in \beta \cap F} W(b \mid \beta)\right)=0$ for every $H \in \mathcal{D}_{k-1} \backslash\{F\}$. Together with $\mu\left(E_{k-1}\right)=1$ one derives $\mu\left(\cdot \mid \bigcup_{b \in \beta \cap F} W(b \mid \beta)\right)=\mu(\cdot \mid F)$.

For $n \in \mathbb{N}$ we call a set $n$-optimal, if it is an $n$-optimal set of order $r$ for $\mu$.

Lemma 4.2 For $k, n \in \mathbb{N}$ let $\beta \subset E_{k-1}$ have exactly n elements. Then the following implications hold. (i) If there exist $G, H \in \mathcal{D}_{k-1}$ with $\operatorname{card}(\beta \cap G)=0$ and $\operatorname{card}(\beta \cap H)>2$, then $\beta$ is not n-optimal. (ii) If $\beta \cap F \neq \emptyset$ for every $F \in \mathcal{D}_{k-1}$ and if there are $G, H \in \mathcal{D}_{k-1}$ with $\operatorname{card}(\beta \cap G)=1$ and $\operatorname{card}(\beta \cap H)>2$, then $\beta$ is not n-optimal.

Proof. (i) For $k=1$ there is nothing to prove. Now let $k>1$ and let $G, H \in \mathcal{D}_{k-1}$ be as in (i). Set $b_{1}=\min (\beta \cap H), b_{3}=\max (\beta \cap H)$ and choose $b_{2} \in \beta \cap H$ with $b_{1}<b_{2}<b_{3}$. For the midpoint $a$ of $G$ define

$$
\gamma=\left(\beta \backslash\left\{b_{2}\right\}\right) \cup\{a\} .
$$

Like in the proof of [6], Lemma 3.4 we get

$$
\int_{E_{k-1} \backslash(G \cup H)} \min _{b \in \beta}|x-b|^{r} d \mu(x) \geq \int_{E_{k-1} \backslash(G \cup H)} \min _{c \in \gamma}|x-c|^{r} d \mu(x)
$$

Since $\beta \cap G=\emptyset, \beta \subset E_{k-1}$ and $\sup _{j \in \mathbb{N}} c_{j} \leq \frac{1}{3}$ we have

$$
\int_{G} \min _{b \in \beta}|x-b|^{r} d \mu(x) \geq \mu(G)(\operatorname{diam}(G))^{r}=\mu(H)(\operatorname{diam}(H))^{r} .
$$

From the definition of $\gamma$ we obtain

$$
\int_{G} \min _{c \in \gamma}|x-c|^{r} d \mu(x) \leq \int_{G}|x-a|^{r} d \mu(x)<\left(\frac{1}{2} \operatorname{diam}(G)\right)^{r} \mu(G) .
$$

Moreover, we derive

$$
\begin{aligned}
& \int_{H} \min _{c \in \gamma}|x-c|^{r} d \mu(x)-\int_{H} \min _{b \in \beta}|x-b|^{r} d \mu(x) \\
\leq & \int_{\left[b_{1}, b_{3}\right]}\left(\min \left(\left|x-b_{1}\right|,\left|x-b_{3}\right|\right)\right)^{r} d \mu(x) \\
\leq & \mu(H)\left(\frac{1}{2} \operatorname{diam}(H)\right)^{r} .
\end{aligned}
$$

Combining inequalities (34), (35) and (36) yields

$$
\begin{aligned}
& \int_{G} \min _{b \in \beta}|x-b|^{r} d \mu(x) \geq \mu(H)(\operatorname{diam}(H))^{r} \\
\geq & \mu(G)\left(\frac{1}{2} \operatorname{diam}(G)\right)^{r}+\mu(H)\left(\frac{1}{2} \operatorname{diam}(H)\right)^{r} \\
> & \int_{G} \min _{c \in \gamma}|x-c|^{r} d \mu(x)+\int_{H} \min _{c \in \gamma}|x-c|^{r} d \mu(x)-\int_{H} \min _{b \in \beta}|x-b|^{r} d \mu(x) .
\end{aligned}
$$

The combination of (33) and (37) shows, that $\beta$ is not $n$-optimal.

(ii) Again, if $k=1$ there is nothing to prove. So let $k>1$ and $G, H \in \mathcal{D}_{k-1}$ be as in (ii). Set $\left\{b_{0}\right\}=G \cap \beta$. Let $b_{1}, b_{2}, b_{3} \in \beta \cap H$ with $b_{1}<b_{2}<b_{3}$. Let $a_{1}$ and $a_{2}$ be the midpoints of $G_{1}$ and $G_{2}$ and $a_{1}^{\prime}$ and $a_{2}^{\prime}$ the midpoints of $H_{1}$ and $H_{2}$ respectively. Set

$$
\gamma=\left(\beta \backslash\left\{b_{0}, b_{1}, b_{2}, b_{3}\right\}\right) \cup\left\{a_{1}, a_{2}, a_{1}^{\prime}, a_{2}^{\prime}\right\} .
$$


Like in the proof of [6], Lemma 3.5 we get

$$
\int_{E_{k-1} \backslash(G \cup H)} \min _{b \in \beta}|x-b|^{r} d \mu(x)=\int_{E_{k-1} \backslash(G \cup H)} \min _{c \in \gamma}|x-c|^{r} d \mu(x)
$$

By Lemma 4.1 we have

$$
\int_{G} \min _{b \in \beta}|x-b|^{r} d \mu(x)=\int_{G}\left|x-b_{0}\right|^{r} d \mu(x) .
$$

By Corollary 3.3 we know that

$$
\int_{G}\left|x-b_{0}\right|^{r} d \mu(x) \geq \int_{G}|x-a|^{r} d \mu(x),
$$

where $a$ is the midpoint of $G$. It is easy to show that

$$
\int_{G}|x-a|^{r} d \mu(x) \geq \frac{1}{2} \mu(G)(\operatorname{diam}(G))^{r}\left(\left(\frac{1}{2}-c_{k}\right)^{r}+\left(\frac{1}{2}-c_{k} c_{k+1}\right)^{r}\right) .
$$

Combining (39) and (40) with (41) we derive

$$
\int_{G} \min _{b \in \beta}|x-b|^{r} d \mu(x) \geq \frac{1}{2} \mu(G)(\operatorname{diam}(G))^{r}\left(\left(\frac{1}{2}-c_{k}\right)^{r}+\left(\frac{1}{2}-c_{k} c_{k+1}\right)^{r}\right) .
$$

Due to $a_{1}, a_{2} \in \gamma$ we obtain

$$
\begin{aligned}
& \int_{G} \min _{c \in \gamma}|x-c|^{r} d \mu(x) \leq \int_{G_{1}}\left|x-a_{1}\right|^{r} d \mu(x)+\int_{G_{2}}\left|x-a_{2}\right|^{r} d \mu(x) \\
\leq & \frac{1}{2} \mu(G)(\operatorname{diam}(G))^{r}\left(\left(\frac{c_{k}}{2}-c_{k} c_{k+1}+c_{k} c_{k+1} c_{k+2}\right)^{r}+\left(\frac{c_{k}}{2}\right)^{r}\right) .
\end{aligned}
$$

In the same way we derive

$$
\int_{H} \min _{c \in \gamma}|x-c|^{r} d \mu(x) \leq \frac{1}{2} \mu(H)(\operatorname{diam}(H))^{r}\left(\left(\frac{c_{k}}{2}-c_{k} c_{k+1}+c_{k} c_{k+1} c_{k+2}\right)^{r}+\left(\frac{c_{k}}{2}\right)^{r}\right) .
$$

Combining (42), (43) and (44) we get

$$
\begin{aligned}
& \int_{G \cup H} \min _{b \in \beta}|x-b|^{r} d \mu(x)-\int_{G \cup H} \min _{c \in \gamma}|x-c|^{r} d \mu(x) . \\
& \geq \frac{1}{2} \mu(G)(\operatorname{diam}(G))^{r}\left[\left(\frac{1}{2}-c_{k}\right)^{r}+\left(\frac{1}{2}-c_{k} c_{k+1}\right)^{r}\right. \\
&\left.-2\left(\frac{c_{k}}{2}-c_{k} c_{k+1}+c_{k} c_{k+1} c_{k+2}\right)^{r}-2\left(\frac{c_{k}}{2}\right)^{r}\right] \\
& \geq \frac{1}{2} \mu(G)(\operatorname{diam}(G))^{r}\left[\left(\frac{1}{2}-c_{k}\right)^{r}+\left(\frac{1}{2}-c_{k} c_{k+1}\right)^{r}\right. \\
&\left.-\left(c_{k}-2 c_{k} c_{k+1}+2 c_{k} c_{k+1} c_{k+2}\right)^{r}-c_{k}^{r}\right] .
\end{aligned}
$$

Now let $A=\frac{1}{2}-c_{k}$ and $B=\frac{1}{2}-c_{k} c_{k+1}$ resp. $C=c_{k}$ and $D=c_{k}-2 c_{k} c_{k+1}+2 c_{k} c_{k+1} c_{k+2}$. Obviously $A, B, C$ and $D$ are non-negative real numbers and we have

$$
A>B>C>D \text {. }
$$

Moreover

$$
\begin{aligned}
& A+B-(C+D)=\frac{1}{2}-c_{k}+\frac{1}{2}-c_{k} c_{k+1}-\left(c_{k}-2 c_{k} c_{k+1}+2 c_{k} c_{k+1} c_{k+2}\right)-c_{k} \\
= & 1-3 c_{k}+c_{k} c_{k+1}\left(1-2 c_{k+2}\right)>1-3 c_{k} \geq 0 .
\end{aligned}
$$


Since $r \geq 1$ we get by Lemma 3.10 that

$$
\left(\frac{1}{2}-c_{k}\right)^{r}+\left(\frac{1}{2}-c_{k} c_{k+1}\right)^{r}-\left(c_{k}-2 c_{k} c_{k+1}+2 c_{k} c_{k+1} c_{k+2}\right)^{r}-c_{k}^{r}>0 .
$$

Combining inequality (47), (46) and (45) we get together with (38)

$$
\int \min _{b \in \beta}|x-b|^{r} d \mu(x)>\int \min _{c \in \gamma}|x-c|^{r} d \mu(x) .
$$

Hence $\beta$ is not $n$ - optimal.

Lemma 4.3 Let $k \in \mathbb{N}_{0}, n \in \mathbb{N}$ and $2^{k} \leq n$. Let $\beta$ be $n$-optimal. Then $\beta \subset E_{k}$ and $F \cap \beta \neq \emptyset$ for every $F \in \mathcal{D}_{k}$.

Proof. 1. Let $k=0$. By [3], Remark 4.6 (a) we know, that $\beta \subset E_{0}$. Since $\mathcal{D}_{0}=\left\{E_{0}\right\}$ we have $F \cap \beta \neq \emptyset$ for every $F \in \mathcal{D}_{0}$.

2. Let $k=1$. Proposition 3.11 yields $\beta \subset E_{1}$ and $(\beta \cap F) \neq \emptyset$ for every $F \in \mathcal{D}_{1}$.

3. Let $k \geq 2$ and assume, that for a $j<k$ we have $\beta \subset E_{j}$. We will show, that $\beta \subset E_{j+1}$ and for every $F \in \mathcal{D}_{j+1}$ we have $\operatorname{card}(\beta \cap F) \geq 1$. Assume, that there is a $G \in \mathcal{D}_{j}$ with $G \cap \beta=\emptyset$. Then we have a $H \in \mathcal{D}_{j}$ with $\operatorname{card}(H \cap \beta)>2$, because otherwise we would get

$$
n=\sum_{F \in \mathcal{D}_{j}} \operatorname{card}(\beta \cap F) \leq 2\left(2^{j}-1\right)<2^{j+1} \leq n,
$$

a contradiction. By Lemma 4.2 (i) this contradicts the optimality of $\beta$. Thus we have $\operatorname{card}(\beta \cap F) \geq 1$ for all $F \in \mathcal{D}_{j}$. Suppose, that a $G \in \mathcal{D}_{j}$ contains only one point. Like in (48) we get a $H \in \mathcal{D}_{j}$ with $\operatorname{card}(H \cap \beta) \geq 3$. By Lemma 4.2 (ii) this contradicts the optimality of $\beta$. Therefore, $\operatorname{card}(\beta \cap F) \geq 2$ for all $F \in \mathcal{D}_{j}$. By [3], Theorem 4.1 we know that for every $F \in \mathcal{D}_{j}$ the set $\beta \cap F$ is an $\operatorname{card}(\beta \cap F)$ - optimal quantizer for $\mu\left(\cdot \mid \bigcup_{b \in \beta \cap F} W(b \mid \beta)\right)$. By Lemma 4.1 we obtain $\mu\left(\cdot \mid \bigcup_{b \in \beta \cap F} W(b \mid \beta)\right)=\mu(\cdot \mid F)$. Hence, Proposition 3.11 and Remark 3.12 yield $\beta \subset E_{j+1}$ and for every $F \in \mathcal{D}_{j+1}$ we have $\operatorname{card}(\beta \cap F) \geq 1$.

4. If one repeats the procedure in 3 . till $j=k-1$, the assertion is proved.

The distribution $\mu$ is defined by the sequence $\left(c_{l}\right)_{l=1}^{\infty}$. For $k \in \mathbb{N}$ let $\mu^{(k)}$ be the dyadic homogeneous Cantor measure defined by the sequence $\left(c_{l+k-1}\right)_{l=1}^{\infty}$. Clearly, $\mu^{(1)}=\mu$. Recall $\pi_{k}=\prod_{i=1}^{k} c_{i}$. Now we can state the main result of this paper.

Theorem 4.4 Let $r>1$ and $n \geq 2$. Let $k \in \mathbb{N}$ and $j \in \mathbb{N}_{0}$ such that $n=2^{k}+j, 0 \leq j<2^{k}$. Let $\beta$ be $n$-optimal. Then $\beta$ consists of the midpoints of some $2^{k}-j$ basic intervals of order $k$ and the midpoints of the $2 j$ basic intervals of order $(k+1)$ contained in the other $j$ basic intervals of order $k$. Moreover,

$$
V_{n, r}(\mu)=\frac{\pi_{k}^{r}}{2^{k}}\left[\left(2^{k}-j\right) V_{1, r}\left(\mu^{(k+1)}\right)+j \cdot c_{k+1}^{r} V_{1, r}\left(\mu^{(k+2)}\right)\right] .
$$

Pro of. By Lemma 4.3 we have $\beta \subset E_{k}$ and $\operatorname{card}(F \cap \beta) \geq 1$ for every $F \in \mathcal{D}_{k}$. Assume, that a $G \in \mathcal{D}_{k}$ exists, with $\operatorname{card}(\beta \cap G)>2$. Due to $n<2^{k+1}$ a $H \in \mathcal{D}_{k}$ exists, with $\operatorname{card}(H \cap \beta)=1$. By Lemma 4.2 (ii) this contradicts the optimality of $\beta$. Hence, for all $F \in \mathcal{D}_{k}$ we get $1 \leq \operatorname{card}(\beta \cap F) \leq 2$. Let $F \in \mathcal{D}_{k}$ be arbitrary. Applying [3], Theorem 4.1 in combination with Lemma 4.1, we obtain, that $\beta \cap F$ is an $\operatorname{card}(\beta \cap F)-$ optimal quantizer of order $r$ for $\mu(\cdot \mid F)$. If $\operatorname{card}(\beta \cap F)=1$, then we get from Corollary 3.3, that $\beta \cap F$ consists of the midpoint of $F$. If $\operatorname{card}(\beta \cap F)=2$, then Lemma 3.6 yields $\operatorname{card}\left(\beta \cap F_{1}\right)=1$ and $\operatorname{card}\left(\beta \cap F_{2}\right)=1$. Like in the proof of Lemma 4.1, one gets in this case that $\mu\left(\cdot \mid F_{i}\right)=\mu\left(\cdot \mid \bigcup_{b \in \beta \cap F_{i}} W(b \mid \beta)\right)$

for every $i \in\{1,2\}$. Applying [3], Theorem 4.1, we obtain that for every $i \in\{1,2\}$ the set $\beta \cap F_{i}$ is an $\operatorname{card}\left(\beta \cap F_{i}\right)$ - optimal quantizer for $\mu\left(\cdot \mid F_{i}\right)$. By Corollary 3.3 we get, that $\beta \cap F_{1}$ consists of the midpoint of $F_{1}$ and $\beta \cap F_{2}$ consists of the midpoint of $F_{2}$. This yields the first part of the assertion. For the optimal quantization error we deduce from the first part that

$$
V_{n, r}(\mu)=\sum_{F \in \mathcal{D}_{k} ; \operatorname{card}(\beta \cap F)=1} \mu(F) V_{1, r}(\mu(\cdot \mid F))+\sum_{F \in \mathcal{D}_{k} ; \operatorname{card}(\beta \cap F)=2} \mu(F) V_{2, r}(\mu(\cdot \mid F)) .
$$


For $l \in \mathbb{N}$ let

$$
\mathbb{R} \ni x \stackrel{H_{l}}{\rightarrow} x \cdot \pi_{l} \in \mathbb{R}
$$

From the construction of $\mu$ we get $\mu\left(\cdot \mid H_{k}([0,1])\right)=\mu^{(k+1)} \circ H_{k}^{-1}$. By Lemma 2.1 we have

$$
\mu(\cdot \mid F)=\mu\left(\cdot \mid H_{k}([0,1])\right) \circ \Theta_{\min (F)}^{-1}
$$

for every $F \in \mathcal{D}_{k}$. By [3], Lemma 3.2 (a) we obtain

$$
V_{1, r}(\mu(\cdot \mid F))=V_{1, r}\left(\mu^{(k+1)} \circ H_{k}^{-1}\right)=\pi_{k}^{r} V_{1, r}\left(\mu^{(k+1)}\right)
$$

for every $F \in \mathcal{D}_{k}$. Further note, that

$$
\begin{aligned}
& V_{2, r}(\mu(\cdot \mid F))=\frac{1}{2} V_{1, r}\left(\mu\left(\cdot \mid F_{1}\right)\right)+\frac{1}{2} V_{1, r}\left(\mu\left(\cdot \mid F_{2}\right)\right) \\
& =V_{1, r}\left(\mu\left(\cdot \mid F_{1}\right)\right)=V_{1, r}\left(\mu^{(k+2)} \circ H_{k+1}^{-1}\right)=\pi_{k+1}^{r} V_{1, r}\left(\mu^{(k+2)}\right)
\end{aligned}
$$

for every $F \in \mathcal{D}_{k}$. Using (51) and (52) we derive from equation (50), that

$$
\begin{aligned}
V_{n, r}(\mu) & =\frac{1}{2^{k}}\left[\left(2^{k}-j\right) \pi_{k}^{r} V_{1, r}\left(\mu^{(k+1)}\right)+j \pi_{k+1}^{r} V_{1, r}\left(\mu^{(k+2)}\right)\right] \\
& =\frac{\pi_{k}^{r}}{2^{k}}\left[\left(2^{k}-j\right) V_{1, r}\left(\mu^{(k+1)}\right)+j \cdot c_{k+1}^{r} V_{1, r}\left(\mu^{(k+2)}\right)\right]
\end{aligned}
$$

and Theorem 4.4 is proved.

Remark 4.5 For $\sup _{k \in \mathbb{N}} c_{k} \leq \frac{1}{4}$, the first part of Theorem 4.4 was proved by Kesseböhmer and Zhu in [6], Proposition 3.6.

Remark 4.6 For $\sup _{k \in \mathbb{N}} c_{k} \leq \frac{1}{4}$ and $r>1$, Kesseböhmer and Zhu (cf. [6], Remark 3.8 (1)) mentioned, that $C_{n, r}(\mu) \subset C_{n, 1}(\mu)$, but $C_{n, 1}(\mu) \backslash C_{n, r}(\mu) \neq \emptyset$. By checking the proof of Theorem 4.4 it is easy to see, that this fact also holds under the weaker assumption $\sup _{k \in \mathbb{N}} c_{k} \leq \frac{1}{3}$.

Although it seems, that no explicit reference exists for the next result, Kesseböhmer and Zhu derived similar inequalities for $\sup _{j \in \mathbb{N}} c_{j} \leq \frac{1}{4}$ (cf. Proof of Theorem 1.6 (3) in [6]).

Corollary 4.7 Let $k, n \in \mathbb{N}$ with $2^{k} \leq n<2^{k+1}$. Then

$$
2^{-k}\left[2^{k+1}-n+\left(n-2^{k}\right) c_{k+1}^{r}\right]\left(\frac{1}{6}\right)^{r} \pi_{k}^{r}<V_{n, r}(\mu)<\left(\frac{1}{2}\right)^{r} \pi_{k}^{r} .
$$

Proof. Since $\sup _{j \in \mathbb{N}} c_{j} \leq \frac{1}{3}$, we obtain for every $l \in \mathbb{N}$ that $\left(\frac{1}{6}\right)^{r}<V_{1, r}\left(\mu^{(l)}\right)<\left(\frac{1}{2}\right)^{r}$. Then, inequality (53) is an easy consequence of equation (49).

\section{Quantization dimension and quantization coefficient}

In this section we will prove a characterization for the existence of the quantization dimension. Moreover, if the quantization dimension exists, we will prove under weak assumptions, that the quantization coefficient does not exist.

The lower resp. upper quantization dimension of $\mu$ of order $r$ is defined as

$$
\underline{D}_{r}(\mu):=\liminf _{n \rightarrow \infty} \frac{r \log (n)}{-\log \left(V_{n, r}(\mu)\right)}
$$

resp.

$$
\bar{D}_{r}(\mu):=\lim \sup _{n \rightarrow \infty} \frac{r \log (n)}{-\log \left(V_{n, r}(\mu)\right)} .
$$


If the two numbers agree, $D_{r}(\mu)=\underline{D}_{r}(\mu)=\bar{D}_{r}(\mu)$ is called the quantization dimension of $\mu$ of order $r$. From [3], Theorem 12.18 one gets, that the quantization dimension always exist for so-called regular probabilities of dimension $D$, where $D=D_{r}$. For example the classical Cantor distribution is regular of $\operatorname{dimension} D_{r}=\frac{\log (2)}{\log (3)}$ (cf. [3], Example 12.10).

For general dyadic homogeneous Cantor measures, the quantization dimension need not exist. This was first shown by Lindsay [7], Example 5.5 (see also [6], Theorem 1.5 (1)). Under the condition $\sup _{j \in \mathbb{N}} c_{j} \leq \frac{1}{4}$ Kesseböhmer and Zhu (cf. [6], Theorem 1.6 (3)) proved a characterization for the existence of the quantization dimension. Moreover they showed for $\sup _{j \in \mathbb{N}} c_{j} \leq \frac{1}{2}$, that

$$
\bar{D}_{r}(\mu)=\lim \sup _{k \rightarrow \infty} \frac{\log (2)}{-\frac{1}{k} \sum_{i=1}^{k} \log \left(c_{i}\right)}
$$

(cf. [6], Theorem $1.6(1))$, resp.

$$
\underline{D}_{r}(\mu) \leq \liminf _{k \rightarrow \infty} \frac{\log (2)}{-\frac{1}{k} \sum_{i=1}^{k} \log \left(c_{i}\right)}
$$

(cf. [6], Proposition 3.1).

In the following proposition we will sharpen their results under the condition $\sup _{j \in \mathbb{N}} c_{j} \leq \frac{1}{3}$.

\section{Proposition 5.1}

$$
\underline{D}_{r}(\mu)=\liminf _{k \rightarrow \infty} \frac{\log (2)}{-\frac{1}{k} \sum_{i=1}^{k} \log \left(c_{i}\right)} \leq \limsup _{k \rightarrow \infty} \frac{\log (2)}{-\frac{1}{k} \sum_{i=1}^{k} \log \left(c_{i}\right)}=\bar{D}_{r}(\mu)
$$

Pro of. Due to (54) and (55) it remains to show, that

$$
\underline{D}_{r}(\mu) \geq \liminf _{k \rightarrow \infty} \frac{\log (2)}{-\frac{1}{k} \sum_{i=1}^{k} \log \left(c_{i}\right)} .
$$

For $n \in \mathbb{N}$ let $k(n) \in \mathbb{N}_{0}, j(n) \in\left[0,2^{k(n)}\left[\right.\right.$ with $n=2^{k(n)}+j(n)$. Since $\left(V_{n, r}(\mu)\right)_{n \in \mathbb{N}}$ is decreasing, we obtain

$$
\begin{aligned}
\underline{D}_{r}(\mu) & =\liminf _{n \rightarrow \infty} \frac{\log \left(2^{k(n)}+j(n)\right)}{-\frac{1}{r} \log \left(V_{2^{k(n)}+j(n), r}(\mu)\right)} \geq \liminf \log _{k \rightarrow \infty} \frac{\log \left(2^{k}\right)}{-\frac{1}{r} \log \left(V_{2^{k+1}, r}(\mu)\right)} \\
& =\liminf _{k \rightarrow \infty}\left(\frac{\log \left(2^{k+1}\right)}{-\frac{1}{r} \log \left(V_{2^{k+1}, r}(\mu)\right)}+\frac{\log \left(\frac{1}{2}\right)}{-\frac{1}{r} \log \left(V_{2^{k+1}, r}(\mu)\right)}\right) .
\end{aligned}
$$

Note, that $\lim _{n \rightarrow \infty} V_{n, r}(\mu)=0$ (cf. [3], Lemma 6.1). This yields

$$
\underline{D}_{r}(\mu) \geq \liminf _{k \rightarrow \infty} \frac{\log \left(2^{k}\right)}{-\frac{1}{r} \log \left(V_{2^{k}, r}(\mu)\right)} .
$$

Using Corollary 4.7, it is straightforward to check, that

$$
\liminf _{k \rightarrow \infty} \frac{\log \left(2^{k}\right)}{-\frac{1}{r} \log \left(V_{2^{k}, r}(\mu)\right)}=\liminf _{k \rightarrow \infty} \frac{\log (2)}{-\frac{1}{k} \sum_{i=1}^{k} \log \left(c_{i}\right)} .
$$

The combination of (57) and (58) proves inequality (56).

Remark 5.2 As an immediate consequence of Proposition 5.1 one gets, that the quantization dimension $D_{r}(\mu)$ exists, if and only if $\left(\frac{1}{k} \sum_{i=1}^{k} \log \left(c_{i}\right)\right)_{k \in \mathbb{N}}$ converges in $\mathbb{R}$. If one of these equivalent conditions is satisfied, then

$$
D_{r}(\mu)=\lim _{k \rightarrow \infty} \frac{\log (2)}{-\frac{1}{k} \sum_{i=1}^{k} \log \left(c_{i}\right)} .
$$


If $D_{r}=D_{r}(\mu)$ exists and $\left(n^{\frac{r}{D_{r}}} V_{n, r}(\mu)\right)_{n \in \mathbb{N}}$ converges towards a value in $] 0, \infty\left[\right.$, we call $\lim _{n \rightarrow \infty} n^{\frac{r}{D_{r}}} V_{n, r}(\mu)$ the $r$-th quantization coefficient of the distribution $\mu$. Next we will show, that under weak assumptions this sequence does not converge, i.e. the quantization coefficient does not exist.

Proposition 5.3 Suppose $\left(\frac{1}{k} \sum_{i=1}^{k} \log \left(c_{i}\right)\right)_{k \in \mathbb{N}}$ converges in $\mathbb{R}$. Then $D_{r}$ exists and

(i) $\limsup _{n \rightarrow \infty} n^{\frac{r}{D_{r}}} V_{n, r}(\mu)<\infty$, if and only if $\lim \sup _{k \rightarrow \infty} 2^{\frac{k}{D_{r}}} \pi_{k}<\infty$

(ii) $\liminf _{n \rightarrow \infty} n^{\frac{r}{D_{r}}} V_{n, r}(\mu)>0$, if and only if $\liminf _{k \rightarrow \infty} 2^{\frac{k}{D_{r}}} \pi_{k}>0$

(iii) if $0<\liminf _{k \rightarrow \infty} 2^{\frac{k}{D_{r}}} \pi_{k}$ and $\lim \sup _{k \rightarrow \infty} 2^{\frac{k}{D_{r}}} \pi_{k}<\infty$, then

$$
0<\liminf _{n \rightarrow \infty} n^{\frac{r}{D_{r}}} V_{n, r}(\mu)<\limsup _{n \rightarrow \infty} n^{\frac{r}{D_{r}}} V_{n, r}(\mu)<\infty .
$$

Pro o f. By Remark 5.2 we know that $D_{r}$ exists. (i) and (ii) follow immediately from Corollary 4.7. (iii) Let $k \geq 2$ and $n_{k}=2^{k}$ resp. $m_{k}=\left(\frac{5}{4}\right) 2^{k}$. Applying (49) we get

$$
n_{k}^{\frac{r}{D_{r}}} V_{n_{k}, r}(\mu)=\left(2^{k}\right)^{\frac{r}{D_{r}}} \pi_{k}^{r} V_{1, r}\left(\mu^{(k+1)}\right)
$$

resp.

$$
\begin{aligned}
m_{k}^{\frac{r}{D_{r}}} V_{m_{k}, r}(\mu) & \geq\left(\left(\frac{5}{4}\right) 2^{k}\right)^{\frac{r}{D_{r}}} \frac{1}{2^{k}}\left[\left(2^{k+1}-\left(\frac{5}{4}\right) 2^{k}\right) \pi_{k}^{r} V_{1, r}\left(\mu^{(k+1)}\right)\right] \\
& =\frac{3}{4}\left(\frac{5}{4}\right)^{\frac{r}{D_{r}}}\left(2^{k}\right)^{\frac{r}{D_{r}}} \pi_{k}^{r} V_{1, r}\left(\mu^{(k+1)}\right) .
\end{aligned}
$$

Note, that

$$
\frac{r}{D_{r}}=\frac{r \lim _{k \rightarrow \infty}\left(-\frac{1}{k} \sum_{i=1}^{k} \log \left(c_{i}\right)\right)}{\log (2)} \geq \frac{\log (3)}{\log (2)} .
$$

Combining this with (60) and (61) we deduce

$$
\frac{m_{k}^{\frac{r}{D_{r}}} V_{m_{k}, r}(\mu)}{n_{k}^{\frac{r}{D_{r}}} V_{n_{k}, r}(\mu)} \geq \frac{3}{4}\left(\frac{5}{4}\right)^{\frac{r}{D_{r}}} \geq \frac{3}{4}\left(\frac{5}{4}\right)^{\frac{\log (3)}{\log (2)}}>1 .
$$

Due to the assumptions in (iii) the combination of (62) with (i) and (ii) yields

$$
\begin{aligned}
0 & <\liminf _{n \rightarrow \infty} n^{\frac{r}{D_{r}}} V_{n, r}(\mu) \leq \liminf _{k \rightarrow \infty} n_{k}^{\frac{r}{D_{r}}} V_{n_{k}, r}(\mu) \\
& <\limsup _{k \rightarrow \infty} m_{k}^{\frac{r}{D_{r}}} V_{m_{k}, r}(\mu) \leq \limsup _{n \rightarrow \infty} n^{\frac{r}{D_{r}}} V_{n, r}(\mu)<\infty .
\end{aligned}
$$

Thus (iii) is proved.

Remark 5.4 Clearly (59) holds, if $\left.\left.c_{i}=c \in\right] 0, \frac{1}{3}\right]$ for every $i \in \mathbb{N}$. It remains an open question, if $\liminf _{n \rightarrow \infty} n^{\frac{r}{D_{r}}} V_{n, r}(\mu)<\lim \sup _{n \rightarrow \infty} n^{\frac{r}{D_{r}}} V_{n, r}(\mu)$ still holds, if we drop some of the assumptions in Proposition 5.3 (iii).

\section{The self-similar case}

If $\left.c_{k}=c \in\right] 0, \frac{1}{2}$ ] for every $k \in \mathbb{N}$, the dyadic homogeneous Cantor distribution $\mu$ becomes self-similiar with contracting parameter $c$. Graf and Luschgy have shown for this type of measure, that the quantization dimension $D_{r}(\mu)$ equals $D=D(c)=\frac{\log (2)}{-\log (c)}$ for every $r>0$ (cf. [4], Remark 5.13(a)).

In this section we obtain for $\left.c \in] 0, \frac{1}{3}\right]$ an explicit formula for the quantization error and can characterize the set of all accumulation points of the sequence $\left(n^{\frac{r}{D}} V_{n, r}(\mu)\right)_{n \in \mathbb{N}}$. 
Remark 6.1 If $\mu$ denotes a self-similar Cantor distribution with contracting parameter $\left.c \in] 0, \frac{1}{3}\right]$, then we have $\mu^{(k)}=\mu$ for all $k$ and, therefore, we obtain

$$
V_{n, r}(\mu)=\left(\frac{c^{r}}{2}\right)^{k} V_{1, r}(\mu)\left(c^{r}\left(n-2^{k}\right)+2^{k+1}-n\right)
$$

as an immediate consequence of Theorem 4.4. With the mapping $f_{c}:[1,2] \rightarrow \mathbb{R}$ defined by

$$
f_{c}(x)=V_{1, r}(\mu) x^{\frac{r}{D}}\left(\left(2-c^{r}\right)-x\left(1-c^{r}\right)\right)
$$

one gets as in the proof of [2], Theorem 6.3, that $f_{c}([1,2])$ equals the set of all accumulation points of the sequence $\left(n^{\frac{r}{D}} V_{n, r}(\mu)\right)_{n \in \mathbb{N}}$. It is easy to see, that $f_{c}(1)=V_{1, r}(\mu)=f_{c}(2)$ and that $f_{c}^{\prime}$ has one critical point

$$
\left.x_{0}:=x_{0}(c):=\frac{2-c^{r}}{\left(1-c^{r}\right)\left(1+\frac{D}{r}\right)} \in\right] 1,2[.
$$

Moreover $f_{c}^{\prime}(1)>0$ resp. $f_{c}^{\prime}(2)<0$. Hence $f_{c}$ attains its maximum at $x_{0}$ and we have $f_{c}([1,2])=\left[f_{c}(1), f_{c}\left(x_{0}\right)\right]=\left[V_{1, r}(\mu), f_{c}\left(x_{0}\right)\right]$. Clearly, the quantization coefficient $\lim _{n \rightarrow \infty} n^{\frac{r}{D}} V_{n, r}(\mu)$ does not exist.

Remark 6.2 As a special case, Remark 6.1 yields the optimal quantization for the classical Cantor Distribution $\left(c_{k}=\frac{1}{3}\right.$ for all $k$ ). For this distribution and $r=2$, the results outlined in Remark 6.1 were first proved in [2] (Theorem 5.2. and Theorem 6.3). Theorem 5.2. in [2] also describes the $n$-optimal sets of order 2 , and, in this respect, is a special case of Theorem 4.4.

Example 6.3 If we drop the assumption $c \leq \frac{1}{3}$, Remark 6.1 resp. Theorem 4.4 becomes wrong. For example, let $\mu$ be self-similar with a contracting parameter $c \in] \frac{5-\sqrt{17}}{2}, \frac{1}{2}\left[\right.$ and $r=2$. Let $F=[0,1]$ and $\alpha=\left\{a_{1}, a_{21}, a_{22}\right\}$ with $a_{1}$ the midpoint of $F_{1}$ and $a_{21}, a_{22}$ the midpoints of $F_{21}, F_{22}$. Assume, that $\alpha \in C_{3,2}(\mu)$. Since $c>\frac{5-\sqrt{17}}{2}$ one can prove that

$$
\mu\left(W\left(a_{21} \mid \alpha\right) \cap F_{1}\right)>0 .
$$

By [3], Theorem 4.1 we obtain $\left\{a_{21}\right\} \in C_{1,2}\left(\mu\left(\cdot \mid W\left(a_{21} \mid \alpha\right)\right)\right)$ and hence

$$
a_{21}=E\left(\mu\left(\cdot \mid W\left(a_{21} \mid \alpha\right)\right)\right) .
$$

By easy computations one can show, that the expected value of $\mu\left(\cdot \mid F_{2} \cap W\left(a_{21} \mid \alpha\right)\right)$ equals $a_{21}$, which differs from $E\left(\mu\left(\cdot \mid W\left(a_{21} \mid \alpha\right)\right)\right)$, due to equation (65). This contradicts equation (66). Hence we have $\alpha \notin C_{3,2}(\mu)$ and Theorem 4.4 is not true.

Remark 6.4 Let $\mu=\mu_{c}$ be self-similar with contracting parameter $\left.\left.c \in\right] 0, \frac{1}{2}\right]$ and $x_{0}(c)$ as defined in (64). If $\left.c \in] 0, \frac{1}{3}\right]$ we know from Remark 6.1, that

$$
\liminf _{n \rightarrow \infty} n^{\frac{r}{D}} V_{n, r}\left(\mu_{c}\right)=V_{1, r}\left(\mu_{c}\right)
$$

and

$$
\lim \sup _{n \rightarrow \infty} n^{\frac{r}{D}} V_{n, r}\left(\mu_{c}\right)=f_{c}\left(x_{0}(c)\right) .
$$

Using the representations (67) and (68), one can show that the mappings

$c \rightarrow \liminf _{n \rightarrow \infty} n^{\frac{r}{D}} V_{n, r}\left(\mu_{c}\right)$ and $c \rightarrow \limsup _{n \rightarrow \infty} n^{\frac{r}{D}} V_{n, r}\left(\mu_{c}\right)$ are continuous on $\left.] 0, \frac{1}{3}\right]$. It is easy to prove, that

$$
\lim _{c \rightarrow 0} f_{c}\left(x_{0}(c)\right)=\lim _{c \rightarrow 0} \limsup _{n \rightarrow \infty} n^{\frac{r}{D}} V_{n, r}\left(\mu_{c}\right)=\infty .
$$


The mapping $c \rightarrow f_{c}\left(x_{0}(c)\right)$ is also defined for $\left.\left.c \in\right] \frac{1}{3}, \frac{1}{2}\right]$. Moreover, one can show, that

$$
\lim _{c \rightarrow \frac{1}{2}-0}\left[f_{c}\left(x_{0}(c)\right)-V_{1, r}\left(\mu_{c}\right)\right]>0 .
$$

If $c=\frac{1}{2}$ we have $D=1$ and $\mu_{\frac{1}{2}}$ equals the uniform distribution on $[0,1]$. The sequence $\left(n^{r} V_{n, r}\left(\mu_{\frac{1}{2}}\right)\right)_{n \in \mathbb{N}}$ converges in this case (see [3], Theorem 6.2 resp. Example 5.5). Hence we know that

$$
\lim _{c \rightarrow \frac{1}{2}-0}\left[f_{c}\left(x_{0}(c)\right)-V_{1, r}\left(\mu_{c}\right)\right]>\lim \sup _{n \rightarrow \infty} n^{r} V_{n, r}\left(\mu_{\frac{1}{2}}\right)-\liminf _{n \rightarrow \infty} n^{r} V_{n, r}\left(\mu_{\frac{1}{2}}\right)=0
$$

showing that the set of all accumulation points of the sequence $\left(n^{\frac{r}{D}} V_{n, r}(\mu)\right)_{n \in \mathbb{N}}$ at $c=\frac{1}{2}$ does not equal the interval $\left[V_{1, r}(\mu), f_{c}\left(x_{0}\right)\right]$ any more. The following questions remain open:

(i) For which values of $\left.c \in] \frac{1}{3}, \frac{1}{2}\right]$, does equation (67) resp. (68) hold, if any ?

(ii) Is $c \rightarrow \liminf _{n \rightarrow \infty} n^{\frac{r}{D}} V_{n, r}\left(\mu_{c}\right)$ resp. $c \rightarrow \limsup _{n \rightarrow \infty} n^{\frac{r}{D}} V_{n, r}\left(\mu_{c}\right)$ continuous on $\left[\frac{1}{3}, \frac{1}{2}\right]$ ?

(iii) If $c \rightarrow \limsup _{n \rightarrow \infty} n^{\frac{r}{D}} V_{n, r}\left(\mu_{c}\right)-\liminf _{n \rightarrow \infty} n^{\frac{r}{D}} V_{n, r}\left(\mu_{c}\right)$ is not continuous at $c=\frac{1}{2}$ what is

$$
\lim _{c \rightarrow \frac{1}{2}-0}\left[\limsup _{n \rightarrow \infty} n^{\frac{r}{D}} V_{n, r}\left(\mu_{c}\right)-\liminf _{n \rightarrow \infty} n^{\frac{r}{D}} V_{n, r}\left(\mu_{c}\right)\right],
$$

provided it exists ?

Acknowledgements I would like to thank the referee for his useful comments.

\section{References}

[1] D.J. Feng, Z.Y. Wen and J. Wu. Some dimensional results for homogeneous Moran sets. Sci. China Ser. A, (40)5 (1997), 475-482.

[2] S. Graf and H. Luschgy. The quantization of the Cantor Distribution. Math. Nachr., 183 (1997), 113-133.

[3] S. Graf and H. Luschgy. Foundations of Quantization for probability distributions, Lecture Notes in Mathematics, 1730, Springer-Verlag, Berlin 2000.

[4] S. Graf and H. Luschgy. Quantization for probability measures with respect to the geometric mean error. Math. Proc. Camb. Phil. Soc. 136 (2004), 687-717.

[5] R. Gray and D. Neuhoff. Quantization. IEEE Trans. Inform. Theory, 44 (1998), 2325-2383.

[6] M. Kesseböhmer and S. Zhu. Stability of quantization dimension and quantization for homogeneous Cantor measure. preprint (2004).

[7] L.J. Lindsay. Quantization dimension for Probability Distributions. Doctoral thesis, Univ. of North Texas, Denton 2001.

[8] L.J. Lindsay and R.D. Mauldin. Quantization dimension for conformal iterated function systems. Nonlinearity, 15(1) (2002), 189-199.

[9] K. Pötzelberger. The quantization error of self-similar distributions. Math. Proc. Camb. Phil. Soc. 137 (2004), $725-740$.

[10] Z.Y. Wen. Mathematical foundations of fractal geometry. Shanghai Scientific and Technological Education Publishing House, 2000.

[11] P.L. Zador. Asymptotic quantization error of continuous signals and the quantization dimension. IEEE Trans. Inform. Theory, 28 (1982), 139-149.

This is a preprint of an article accepted for publication in Mathematische Nachrichten,

Print ISSN: 0025-584X, Online ISSN: 1522-2616,

Copyright (C)2000-2007 by John Wiley \& Sons, Inc. or related companies.

URL: http://www3.interscience.wiley.com/cgi-bin/jhome/60500208 